Bulletin of the Section of Logic

Volume 45:3/4 (2016), pp. 239-256

http://dx.doi.org/10.18778/0138-0680.45.3.4.07

Adam Kolany* and Miroslaw Wrobel

\title{
SOME ALGEBRAIC AND ALGORITHMIC PROBLEMS IN ACOUSTOCEREBROGRAPHY
}

\begin{abstract}
Progress in the medical diagnostic is relentlessly pushing the measurement technology as well with its intertwined mathematical models and solutions. Mathematics has applications to many problems that are vital to human health but not for all. In this article we describe how the mathematics of acoustocerebrography has become one of the most important applications of mathematics to the problems of brain monitoring as well we will show some algebraic problems which still have to be solved. Acoustocerebrography $([4,1])$ is a set of techniques of visualizing the state of (human) brain tissue and its changes with use of ultrasounds, which mainly rely on a relation between the tissue density and speed of propagation for ultrasound waves in this medium. Propagation speed or, equivalently, times of arriving for an ultrasound pulse, can be inferred from phase relations for various frequencies. Since, due to Kramers-Kronig relations, the propagation speeds depend significantly on the frequency of investigated waves, we consider multispectral wave packages of the form $\mathcal{W}(n)=$ $\sum_{h=1}^{H} A_{h} \cdot \sin \left(2 \pi \cdot f_{h} \cdot n / F+\psi_{h}\right), n=0, \ldots, N-1$, with appropriately chosen frequencies $f_{h}, h=1, \ldots, H$, amplifications $A_{h}, h=1, \ldots, H$, start phas̄es $\psi_{h}, h=1, \ldots, H$, and sampling frequency $F$. In this paper we show some problems of algebraic and, to some extend, algorithmic nature which raise up in this topic. Like, for instance, the influence of relations between the signal length and frequency values on the error on estimated phases or on neutralizing alien frequencies. Another problem is finding appropriate initial phases for avoiding
\end{abstract}

\footnotetext{
*Corresponding author: Adam Kolany, Sonovum A.G., Perlickstrasse 5, 04103 Leipzig, Germany
} 
improper distributions of peaks in the resulting signal or finding a stable algorithm of phase unwinding which is resistant to sudden random disruptions.

Keywords: ACG, Acoustocerebrography, Stroke, Brain Monitoring, Neurology, Signal processing, multispectral signal decomposition, matrix condition, error estimation, phase unwinding

\section{Introduction}

Progress in the medical diagnostic is relentlessly pushing the measurement technology as well with its intertwined mathematical models and solutions. Mathematics has applications to many problems that are vital to human health but not for all. In this article we describe how the mathematics of acoustocerebrography has become one of the most important applications of mathematics to the problems of brain monitoring as well we will show some algebraic problems which still have to be solved. Acoustocerebrography is a set of techniques of visualizing the state of (human) brain tissue and its changes with use of ultrasounds. A particular stress is put onto the changes resulting from brain stroke. The changes of this kind are highly related to the changes of tissue density $(\varrho)$ which, in turn, reflects in changes of sound velocity $(c)$ according to the formula (in the simplest model): $c \sim \sqrt{1 / \varrho}$. In order to estimate the speed of a sound wave traveling through the humans brain tissue we use two piezoelectric transducers placed at opposite locations ${ }^{1}$ of a human skull (Figure 1.1a), emit a short $(\sim 100 \mu \mathrm{s})$ ultrasound sampled pulse by one of them and receive it by the other one (see Figure 1.1b). In this paper we show some problems of algebraic and, to some extend, algorithmic nature which raise up in this topic.

Given a distance $(\ell)$ which a wave has to propagate and the time $\left(T^{(\text {Arr) }}\right)$ a wave forehead needs to arrive to the receiver, one could easily compute the velocity as $c=\ell / T^{\text {(Arr) }}$. The problem however is that the time $T^{\text {(Arr) }}$ cannot be properly estimated from just observing the received values because of their severe distortions (see Figure 1.4). Hence one of the possibilities is to fix a time position $\left(T_{X}>T^{(\mathrm{Arr})}\right)$ in the observed signal and try to find the best fitting sinus-like curve in its neighborhood (see Figure 1.2) which can be done with the standard Least Square

\footnotetext{
${ }^{1}$ There are also solutions with both transducers at the same side of the skull. Then the signal which is received is a deflection of the send one off the opposite bones
} 


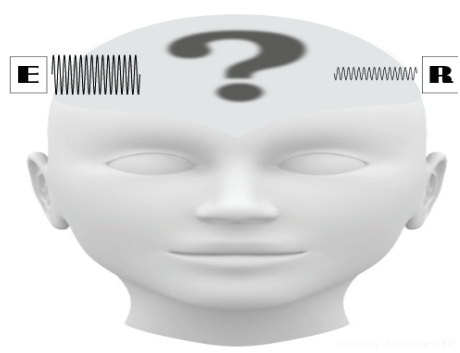

(a) Standard positions of transducers at a human's head
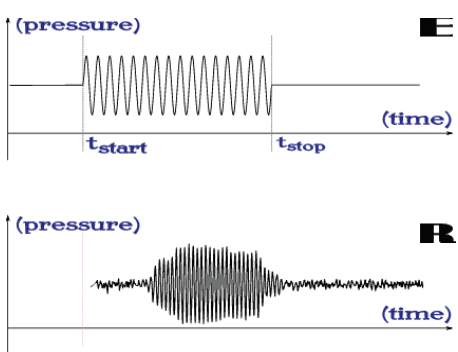

(b) Sent and received signals

Fig. 1.1. Position of transducers and the shapes of the sent signal and a received one.

Method (which algebraically corresponds to projecting of the received signal as a multidimensional vector onto the appropriate subspace, and then to determining the coordinates of the obtained projection in an appropriate basis). Using complex numbers, one can obtain also a more compact formula: $\varphi=\operatorname{Arg}(\mathfrak{Z})$ and $A=|\mathfrak{Z}|$ where $\mathfrak{Z}=\Lambda \cdot Y, Y$ is the column vector consisting of the measured values $Y_{0}, Y_{1}, \ldots, Y_{N-1}$ sampled with the frequency $F$ and $\Lambda$ being the lower row of $2 \imath \cdot\left[\left(\mathcal{B} \cdot \mathcal{B}^{*}\right)^{-1} \cdot \mathcal{B}\right]$ with

$$
\mathcal{B}=\left(\begin{array}{ccccc}
1 & e^{+2 \pi \imath f \cdot 1 / F} & e^{+2 \pi \imath f \cdot 2 / F} & \ldots & e^{+2 \pi \imath f \cdot(N-1) / F} \\
1 & 1 & 1 & \ldots & 1 \\
1 & e^{-2 \pi \imath f \cdot 1 / F} & e^{-2 \pi \imath f \cdot 2 / F} & \ldots & e^{-2 \pi \imath f \cdot(N-1) / F}
\end{array}\right)
$$

As a matter of fact knowing the phase $(\varphi)$ does not determine the time $T^{\text {(Arr) }}$ without knowing the number of wavelengths $(K)$ which fit into $\ell$ (which in turn depend on the velocity) and inspecting the picture 1.3 and performing some simple trigonometric transformations, one obtains only that

$$
2 \pi \cdot f \cdot\left(T_{X}-T^{(\operatorname{Arr})}\right) \equiv \varphi+2 \pi \cdot K
$$

but if one uses the equation 1.2 simultaneously for two frequencies $f_{1}$ and $f_{2}$ satisfying $T_{X} \leqslant \frac{1}{f_{2}-f_{1}}$ (which is a quite reasonable condition) then one gets: 


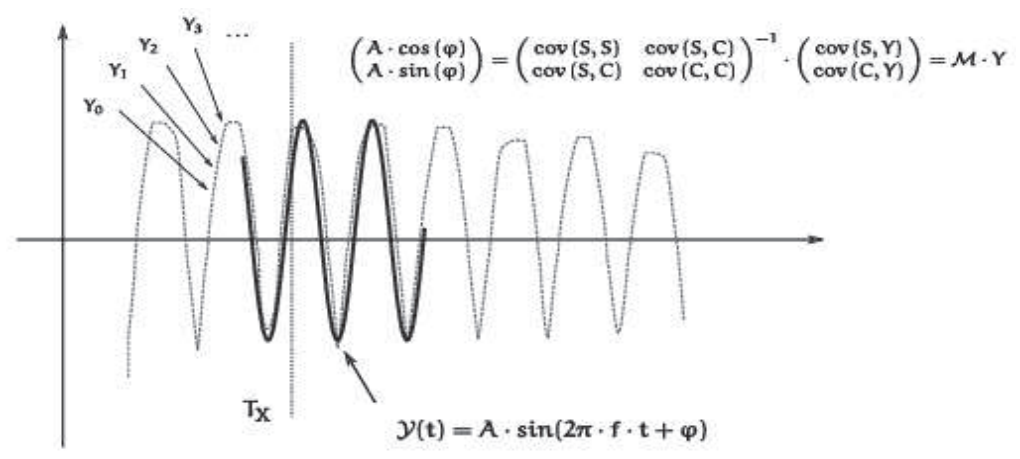

Fig. 1.2. Finding the best fitting sine-like curve.

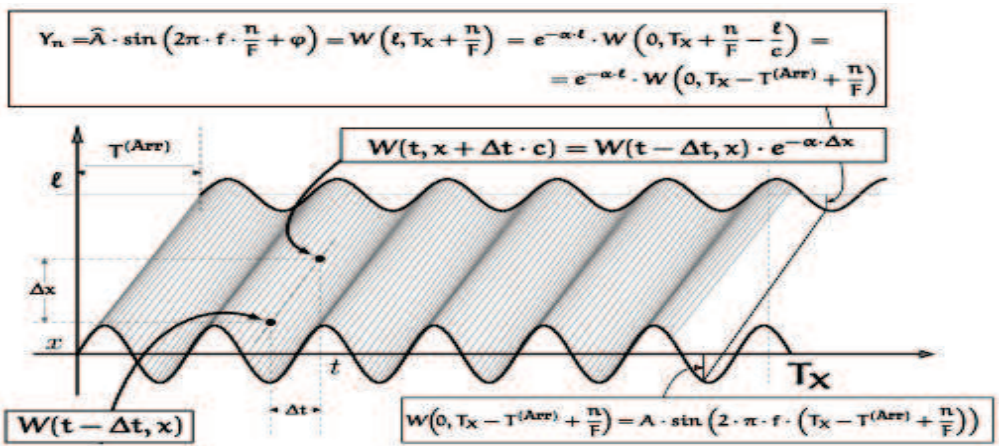

Fig. 1.3. Relating time of arriving with estimated phase

$$
\begin{aligned}
\left(T_{x}-T^{(\mathrm{Arr})}\right) \cdot\left(f_{2}-f_{1}\right) & =\frac{\varphi_{2}-\varphi_{1}}{2 \pi}+\left(K_{2}-K_{1}\right)= \\
= & \left(\left\lfloor\frac{\varphi_{2}-\varphi_{1}}{2 \pi}\right\rfloor+\bmod \left(\frac{\varphi_{2}-\varphi_{1}}{2 \pi}, 1\right)\right)-\left\lfloor\frac{\varphi_{2}-\varphi_{1}}{2 \pi}\right\rfloor= \\
= & \bmod \left(\frac{\varphi_{2}-\varphi_{1}}{2 \pi}, 1\right)=\frac{\bmod \left(\varphi_{2}-\varphi_{1}, 2 \pi\right)}{2 \pi}
\end{aligned}
$$

Hence

$$
T^{(\text {Arr })}=T_{x}-\frac{\bmod \left(\varphi_{2}-\varphi_{1}, 2 \pi\right)}{2 \pi \cdot\left(f_{2}-f_{1}\right)}
$$




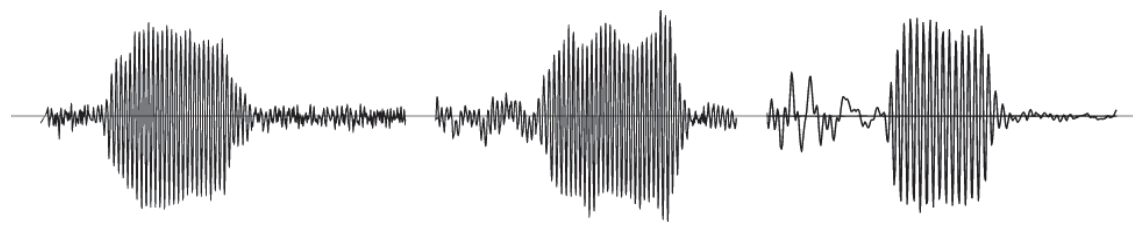

Fig. 1.4. Distortions at beginnings of pulses
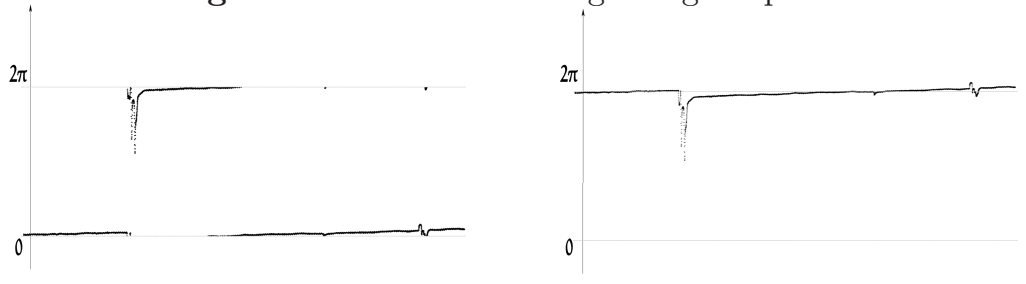

Fig. 1.5. Measured phases before and after unwinding

This attempt, however, requires either sending two pulses with two close frequencies $f_{1}$ and $f_{2}$ simultaneously or one after another with a delay between them short enough so that no biologically essential changes could take place in the meantime. The first attempt actually means that the both pulses sum up into one compound wave of the form:

$$
W(t)=A_{1} \cdot \sin \left(2 \pi \cdot f_{1} \cdot t+\psi_{1}\right)+A_{2} \cdot \sin \left(2 \pi \cdot f_{2} \cdot t+\psi_{2}\right)
$$

with appropriately chosen amplifications $A_{1}$ and $A_{2}$. Then however, estimating the required phases may be bared with quite big inaccuracy since the underlying projection matrix can be poorly conditioned (for details see 2.1). In the other case it can turn out that inaccuracies stemming from rounding projection matrices to the resolution used in the computing software may be essential in relation to the difference $f_{2}-f_{1}$.

Thus one possible solution for observing the evolution/changes of brain tissue properties is to measure phases (and attenuation) many times in a row and trying to recover a possible evolution of times of arriving due to the formula 1.2 with use a kind of "unwinding" procedure (see Figure 1.5 and 2.2 for more details) 


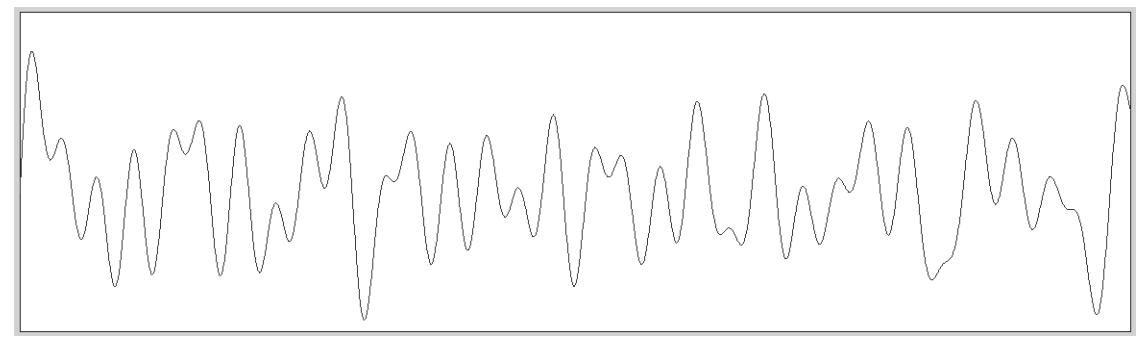

Fig. 1.6. Multispectral signal with $H=8$ frequencies

\section{Multispectrality}

According to the Kramers - Kronig relations (see [2]), the velocity of an ultrasound wave changes with frequency $(f)$ according to the attenuation $(\alpha=\alpha(f))$ :

$$
\frac{1}{c_{2}}-\frac{1}{c_{1}}=-\frac{1}{\pi^{2}} \cdot \int_{f_{1}}^{f_{2}} \frac{\alpha(f) d f}{f^{2}}
$$

That in particular means that the equality 1.3 is not generally valid in a wide range of frequencies and instead we get its generalization ${ }^{2}$ :

$$
\left(f_{2}-f_{1}\right) \cdot\left(T_{X}-\frac{f_{2} / c_{2}-f_{1} / c_{1}}{f_{2}-f_{1}} \cdot \ell\right) \equiv \frac{\varphi_{2}-\varphi_{2}}{2 \pi} \quad(\bmod 1)
$$

for any two frequencies $f_{1}, f_{2}$ with $f_{1}<f_{2}$. Moreover the dependency of wave velocity and frequency suggests that the interrelations between phases for various frequencies may indicate some alarming or at least interesting changes in the investigated tissue.

The above suggests that one should use many frequencies at once composed in one signal: $\mathcal{W}(t)=\sum_{h=1}^{H} A_{h} \cdot \sin \left(2 \pi \cdot f_{h} \cdot t+\psi_{h}\right)$ (see e.g. Figure 1.6). The number $H$ of frequencies which are to be used, their values with relation to the sampling frequency and the values of initial amplifications $A_{1}, \ldots, A_{H}$ and phases $\psi_{1}, \ldots, \psi_{H}$ will be discussed later.

\footnotetext{
${ }^{2} \ell$ is the length of acoustic trace here. That is the distance the wave forehead makes between being sent and being received. In the case of standard positions of transducers, it is the human's head width.
} 


\section{Details}

\subsection{Phase estimating and minimizing the errors}

Let $H>1$ be an integer, $1 \ll F \leqslant 100$ a rational number (the sampling frequency) and $0<f_{1}, \ldots, f_{H} \ll F / 2$ be rational numbers (base frequencies). Let, moreover, $\mathcal{Y}=\left\langle Y_{0}, \ldots, Y_{N-1}\right\rangle$ be a sequence of reals (measured signal). We are concerned with finding such values of $A_{1}, \ldots, A_{h} \geqslant 0$, $\beta \in \mathbb{R}$ and $\varphi_{1}, \ldots, \varphi_{H} \in[0,2 \pi)$ so that the square of the residuum ${ }^{3}$ :

$$
\varepsilon^{2}=\sum_{n=0}^{N-1}\left[Y_{n}-\left(\beta+\sum_{h=1}^{H} A_{h} \cdot \sin \left(2 \pi \cdot f_{h} \cdot n / F+\varphi_{h}\right)\right)\right]^{2}
$$

is as small as possible. This problem can be reformulated as the problem of finding the coordinates $\mathfrak{Z}_{-H}, \mathfrak{Z}_{-H+1}, \ldots, \mathfrak{Z}_{0}, \ldots, \mathfrak{Z}_{H-1}, \mathfrak{Z}_{H} \in \mathbb{C}$ of projection of $Y \in \mathbb{R}^{N}$ onto $2 H+1$ - dimensional space generated by vectors

$$
\mathbf{e}_{-H}, \mathbf{e}_{-H+1}, \ldots, \mathbf{e}_{0}, \ldots, \mathbf{e}_{H-1}, \mathbf{e}_{H}
$$

where

$$
\begin{array}{r}
\mathbf{e}_{h}=\left\langle 1, e^{-2 \imath \pi \cdot \operatorname{sgn}(h) \cdot f_{|h|} \cdot 1 / F}, \ldots, e^{-2 \imath \pi \cdot \operatorname{sgn}(h) \cdot f_{|h|} \cdot(N-1) / F}\right\rangle, \\
h=-H, \ldots, 0, \ldots, H
\end{array}
$$

and $f_{0}=0$. That is such $\mathfrak{Z}_{-H}, \mathfrak{Z}_{-H+1}, \ldots, \mathfrak{Z}_{0},, \ldots, \mathfrak{Z}_{H-1}, \mathfrak{Z}_{H} \in \mathbb{C}$ that

$\left[\begin{array}{c}Y_{0} \\ Y_{1} \\ \vdots \\ \vdots \\ \vdots \\ Y_{N-2} \\ Y_{N-1}\end{array}\right]=\left[\begin{array}{ccccc}1 & \ldots & 1 & \ldots & 1 \\ e^{+\imath \cdot 2 \pi \cdot f_{H} \cdot 1 / F} & \cdots & 1 & \cdots & e^{+\imath \cdot 2 \pi \cdot f_{-H} \cdot 1 / F} \\ \vdots & \vdots & \vdots & \vdots & \vdots \\ \vdots & \vdots & \vdots & \vdots & \vdots \\ e^{+\imath \cdot 2 \pi \cdot f_{H} \cdot \cdot(N-2) / F} & \cdots & 1 & \cdots & e^{+\imath \cdot 2 \pi \cdot f_{-H} \cdot(N-2) / F} \\ e^{+\cdot \cdot 2 \pi \cdot f_{H} \cdot(N-1) / F} & \cdots & 1 & \cdots & e^{+\imath \cdot 2 \pi \cdot f_{-H} \cdot(N-1) / F}\end{array}\right] \cdot\left[\begin{array}{c}\mathfrak{Z}_{-H} \\ \mathfrak{Z}_{-H+1} \\ \vdots \\ \mathfrak{Z}_{0} \\ \vdots \\ \mathfrak{Z}_{H-1} \\ \mathfrak{Z}_{H}\end{array}\right]$

where $f_{0}=0$ and $f_{-h}=-f_{h}, h=1, \ldots, H$. Then

\footnotetext{
${ }^{3}$ technically it is the power of the "noise" in the signal
} 


$$
A_{h}=2 \cdot\left|\mathfrak{Z}_{h}\right|, \quad \varphi_{h}=\operatorname{Arg}\left(\imath \mathfrak{Z}_{h}\right) \in\langle 0,2 \pi), h=1, \ldots, H, \text { and } \beta=2 \cdot \mathfrak{Z}_{0}
$$

As it is known (see e.g. [3]), the coordinates

$$
\mathfrak{Z}=\left[\mathfrak{Z}_{-H}, \mathfrak{Z}_{-H+1}, \ldots, \mathfrak{Z}_{0}, \ldots, \mathfrak{Z}_{H-1}, \mathfrak{Z}_{H}\right]^{\top}
$$

can be obtained as

$$
\mathfrak{Z}=\left(\mathcal{B B}^{*}\right)^{-1} \mathcal{B} \cdot Y
$$

where $\mathcal{B}$ is the conjugate transpose of the matrix to the left hand side of the formula 2.4. Let us also notice that the matrix $\mathcal{M}=\left(\mathcal{B B}^{*}\right)^{-1} \mathcal{B}$ is the Moore-Penrose pseudo-inverse $\left(\mathcal{B}^{\star}\right)^{+}$of $\mathcal{B}^{\star}$ (see [3]). Then also

$$
\mathcal{M}^{+}=\left(\mathcal{B}^{\star}\right)^{++}=\mathcal{B}^{\star}
$$

The main problem which one comes across here is that the measured values $Y$ are never the exact values, which can result from two reasons. The first one is random noise and rounding errors due to digitalizing. Another one can result from "alien" frequencies which may occur in the measured signal for unknown reasons (see 2 for more details).

\section{Estimating phase errors due to random noise and rounding errors}

It can be shown that

Remark 1 Let $u, w \in \mathbb{C}$, let $0<\varepsilon \ll 1$ and let $\varrho=\arcsin (\varepsilon) \approx \varepsilon$. Then

$$
\left|\frac{u-w}{w}\right|<\varepsilon \Rightarrow \operatorname{Arg}\left(u \cdot w^{\star}\right) \in\langle 0, \varrho\rangle \cup\langle 2 \pi-\varrho, 2 \pi\rangle
$$

That is ${ }^{4}$

$$
\operatorname{abs}(\operatorname{Arg}(u)-\operatorname{Arg}(w)) \in\langle 0, \varrho\rangle
$$

which actually means that the arguments of $u$ and $w$ are close.

Now, given a multi-spectral measured signal $Y$ and its error-free counterpart $\tilde{Y}$, the phase of a fixed base frequency $f_{h}(h=1, \ldots, H)$ equals

\footnotetext{
${ }^{4}$ The absolute value, abs $(\alpha)$, of an angle $\alpha$ is ment here as the distance of $\alpha$ to its closest multiplicity of $2 \pi$.
} 
$\varphi_{h}=\operatorname{Arg}\left(\mathfrak{Z}_{h}\right)$ and $\widetilde{\varphi}_{h}=\operatorname{Arg}\left(\widetilde{\mathfrak{Z}}_{h}\right)$, respectively, where $\mathfrak{Z}=\mathcal{M} \cdot Y$ and $\widetilde{\mathfrak{Z}}=\mathcal{M} \cdot \widetilde{Y}$. Then, according to the Remark 1 and inequality ${ }^{5}$

$$
\frac{\|\mathfrak{Z}-\tilde{\mathfrak{Z}}\|}{\|\mathfrak{Z}\|} \leqslant \frac{\operatorname{Cond}(\mathcal{M})}{\cos \vartheta} \cdot \frac{\|Y-\tilde{Y}\|}{\|Y\|}
$$

where $\vartheta$ is the angle between the signal $Y$ and its projection onto the space spanned by the rows of $\mathcal{B}$, we obtain:

$$
\begin{aligned}
& \operatorname{abs}\left(\varphi_{h}-\widetilde{\varphi}_{h}\right) \approx\left|\frac{\mathfrak{Z}_{h}-\widetilde{\mathfrak{Z}}_{h} \mid}{\mathfrak{Z}_{h}}\right|=\frac{\left|\mathfrak{Z}_{h}-\widetilde{\mathfrak{Z}}_{h}\right|}{\left|\mathfrak{Z}_{h}\right|} \leqslant \frac{\|\mathfrak{Z}-\widetilde{\mathfrak{Z}}\|}{\left|\mathfrak{Z}_{h}\right|}= \\
= & \frac{\|\mathfrak{Z}-\widetilde{\mathfrak{Z}}\|}{\|\mathfrak{Z}\| \cdot\left|\mathfrak{Z}_{h}\right| /\|\mathfrak{Z}\|}=\frac{1}{\left|\mathfrak{Z}_{h}\right| /\|\mathfrak{Z}\|} \cdot \frac{\|\mathfrak{Z}-\widetilde{\mathfrak{Z}}\|}{\|\mathfrak{Z}\|}= \\
= & \frac{1}{\varkappa_{h}} \cdot \frac{\operatorname{Cond}(\mathcal{M})}{\cos \vartheta} \cdot \frac{\|Y-\widetilde{Y}\|}{\|Y\|} \leqslant \frac{1}{\varkappa_{h} \cdot \cos \vartheta} \cdot \operatorname{Cond}(\mathcal{M}) \cdot \nu= \\
= & \operatorname{Cond}(\mathcal{M}) \cdot \frac{\nu}{\varkappa_{h} \cdot \cos \vartheta}=\operatorname{Cond}\left(\mathcal{B}^{*}\right) \cdot \frac{\nu}{\varkappa_{h} \cdot \cos \vartheta}
\end{aligned}
$$

where $\varkappa_{h}=\left|\mathfrak{Z}_{h}\right| /\left\|_{\mathfrak{Z}}\right\|$ and $\nu=\|Y-\tilde{Y}\|_{/}\|Y\|$. Hence the error on measured phases depends on the relative error $(\nu)$ of the very measurement, the damping $\left(\varkappa_{h}\right)$ of the investigated frequency in comparison to the others, the relative distance of the signal and its projection (via $\cos (\vartheta)$ ) and the condition of $\mathcal{M}$

$$
\operatorname{Cond}(\mathcal{M})=\|\mathcal{M}\| \cdot\left\|\mathcal{M}^{+}\right\|=\left\|\left(\mathcal{B}^{*}\right)^{+}\right\| \cdot\left\|\mathcal{B}^{*}\right\|=\operatorname{Cond}\left(\mathcal{B}^{*}\right)
$$

which depends on the base frequencies $f_{1}, \ldots, f_{H}$, the sampling frequency $F$ and the number of points, $N$, in the measured signal. Since we have almost no influence on the value of $\nu$, we can only believe that it is relatively small. Then also the value of $\varkappa_{h}$ can be estimated from the measured values and the frequencies whose values of $\varkappa$ are too small as well as the measurements with small values of $\cos \vartheta$ can be just neglected in further considerations. The only thing for which we can influence are the values of base frequencies,

\footnotetext{
${ }^{5}\|\mathfrak{Z}-\widetilde{\mathfrak{Z}}\| \cdot\|Y\| \cdot \cos \vartheta=\|\mathcal{M} \cdot(Y-\tilde{Y})\| \cdot\|Y\| \cdot \frac{\left\|\mathcal{B}^{\star} \mathfrak{Z}\right\|}{\|Y\|} \leqslant\|\mathcal{M}\| \cdot\|Y-\tilde{Y}\| \cdot\left\|\mathcal{B}^{\star}\right\| \cdot\|\mathfrak{Z}\|=$ $\|\mathcal{M}\| \cdot\|Y-\tilde{Y}\| \cdot\left\|\mathcal{M}^{\dagger}\right\| \cdot\|\mathfrak{Z}\|=\operatorname{Cond}(\mathcal{M}) \cdot\|Y-\widetilde{Y}\| \cdot\|\mathfrak{Z}\| \quad \Rightarrow \quad \frac{\|\mathfrak{Z}-\widetilde{\mathfrak{Z}}\|}{\|\mathfrak{Z}\|} \leqslant \frac{\|Y-\tilde{Y}\|}{\|Y\|} \cdot \frac{\operatorname{Cond}(\mathcal{M})}{\cos \vartheta}$
} 


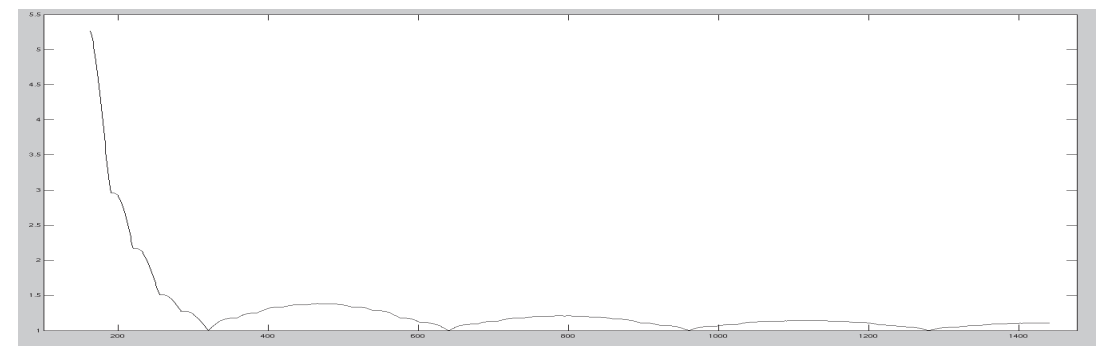

Fig. 2.1. Values of Cond $(\mathcal{M})$ as a function of signal length

the frequency of sampling and the number of values we use for estimating the phases. Hence there arises the following:

Problem 1.

- For a given range of lengths, sampling frequency $F$ and $H$, find (quickly) a set/all sets of frequencies $f_{1}, \ldots, f_{H}$ that $\operatorname{Cond}(\mathcal{M})$ is possibly small.

- Express analytically the correspondence

$$
\left\langle F, f_{1}, \ldots, f_{H}, N\right\rangle \mapsto \operatorname{Cond}(\mathcal{M})
$$

- Find the best analytically expressible upper bound of Cond $(\mathcal{M})$.

Remark 2. The minima of Cond $(\mathcal{M})$, as observed in the Figure 2.1, occur for those $N$ for which $N \cdot f_{h} / F(h=1, \ldots, H)$ are integer. Then the matrix $\mathcal{B} \cdot \mathcal{B}^{*}$ is of the form $N \cdot \mathcal{I}$ where $\mathcal{I}$ is the identity matrix of size $2 H+1$, and then

$$
\operatorname{Cond}(\mathcal{M})=\operatorname{Cond}\left(\mathcal{B}^{\star}\right)=\sqrt{\operatorname{Cond}\left(\mathcal{B} \cdot \mathcal{B}^{\star}\right)}=\sqrt{\operatorname{Cond}((1 / N) \cdot \mathcal{J})}=1 .
$$

\section{Neutralizing "alien" frequencies}

The irregularities of shapes of pulse envelope observed in the Figure 1.4 originate from two sources. First of them is that at the beginning the ultrasound transducer accelerates and needs some time to reach the full power; 
at the end, in turn, the transducer is being switched off and gradually loses its energy. Another source of irregularities in the shape of the pulse's envelope which manifest themselves in the central region of the signal, is the presence of "alien" frequencies. That is, the frequencies which were not present in the original signal. To see this let us, for simplicity, assume that the envelope exhibits presence of one low frequency $G<f$ with amplitude $C<1 / 2$ and the emitted signal was a pulse built up of one frequency $f$ like in the Figure 2.2. Then we have:

$$
\begin{aligned}
& (1+C \cdot \cos (2 \pi \cdot G \cdot t+\psi)) \cdot \sin (2 \pi \cdot f \cdot t+\varphi)= \\
= & \sin (2 \pi \cdot f \cdot t+\varphi)+C \cdot \cos (2 \pi \cdot G \cdot t+\psi) \cdot \sin (2 \pi \cdot f \cdot t+\varphi)= \\
= & \sin (2 \pi \cdot f \cdot t+\varphi)+C_{0} \cdot\left[\sin \left(2 \pi \cdot G_{1} \cdot t+\varphi_{1}\right)+\sin \left(2 \pi \cdot G_{2} \cdot t+\varphi_{2}\right)\right]
\end{aligned}
$$

where $\varphi_{1,2}=\frac{\varphi \pm \psi}{2}, G_{1,2}=\frac{f \pm G}{2}$ and $C_{0}=\frac{C}{2}$. So, as we see, slight "waving" of the envelope manifests itself in the form of two additional frequency components each with amplitude equal to the half of the amplitude of the observed distortion. As a result of this, while computing the phase using $\mathcal{M}$ which is designed to provide information on the base frequencies only, we mistake by some component stemming from the alien frequencies as it is shown below:

$\widehat{\mathfrak{Z}}=\mathcal{M} \cdot(\tilde{Y}+\overbrace{A_{h} \cdot \sin \left(2 \pi \cdot \frac{f_{h}}{F} \cdot \ldots+\varphi_{h}\right)+\cdots}^{Y})=\mathcal{M} \cdot \widetilde{Y}+\mathcal{M} \cdot Y=\widetilde{\mathfrak{Z}}+\mathfrak{Z}$

where $Y$ is the "pure" part of the signal and $\tilde{Y}$ is its "alien part". Then the phase we obtain differs from the valid one by the value (see Figure 2.3) $\delta \varphi_{h}=\operatorname{abs}\left(\operatorname{Arg}\left(\mathfrak{Z}_{h}\right)-\operatorname{Arg}\left(\widehat{\mathfrak{Z}}_{h}\right)\right)$. Hence, by Remark 1 ,

$\delta \varphi_{h} \approx\left|\frac{\widehat{\mathfrak{Z}}_{h}-\mathfrak{Z}_{h}}{\mathfrak{Z}_{h}}\right|=\frac{\left|\widehat{\mathfrak{Z}}_{h}-\mathfrak{Z}_{h}\right|}{\left|\mathfrak{Z}_{h}\right|}=\frac{\left|\widetilde{\mathfrak{Z}}_{h}\right|}{A_{h} / 2} \leqslant \frac{\|\widetilde{\mathfrak{Z}}\|}{A_{h} / 2}=\frac{\|\mathcal{M} \tilde{Y}\|}{A_{h} / 2} \leqslant \frac{2 \cdot\|\mathcal{M}\| \cdot\|\tilde{Y}\|}{A_{h}}$

On the other hand, assuming that the alien part consists of frequency $G$ only, the norm of $\widetilde{Y}$ can be obtained from 

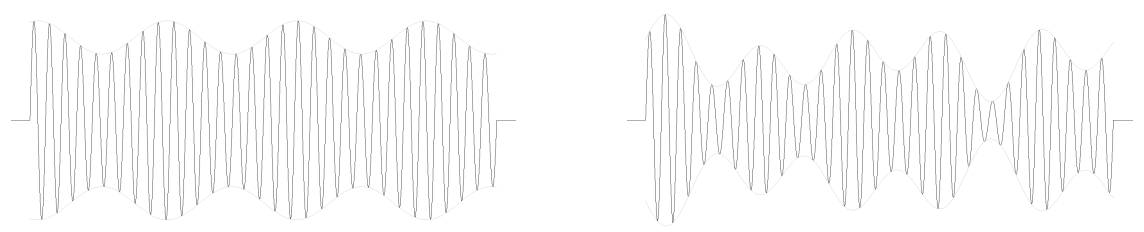

Fig. 2.2. Signal distorted by one (to the left) and three (to the right) alien frequencies

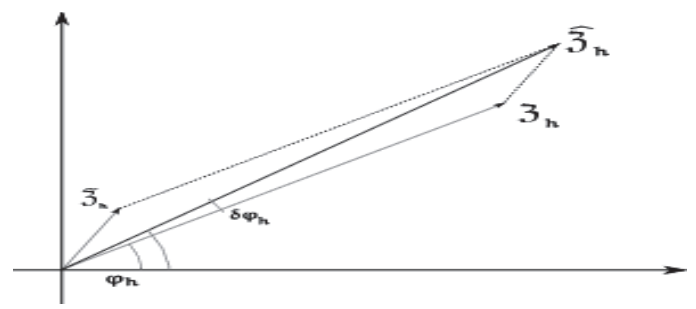

Fig. 2.3. Influence of an alien frequence on the value of a phase

$$
\begin{aligned}
\|\widetilde{Y}\|^{2} & =\sum_{n<N}\left(C \cdot \sin \left(2 \pi \cdot G \cdot \frac{n}{F}+\psi\right)\right)^{2}= \\
& =C^{2} \cdot \sum_{n<N} \sin ^{2}\left(2 \pi \cdot G \cdot \frac{n}{F}+\psi\right) \leqslant N \cdot C^{2}
\end{aligned}
$$

It is easy to notice that given more alien frequencies $G_{1}, \ldots, G_{L}$, with amplitudes $C_{1}, \ldots, C_{L}$, respectively, the above reads

$$
\|\tilde{Y}\|^{2} \leqslant N \cdot \sum_{1 \leqslant l \leqslant L} C_{l}^{2}=N \cdot\|\mathcal{C}\|^{2} .
$$

Hence

$$
\delta \varphi_{h} \leqslant \frac{2 \cdot\|\mathcal{M}\| \cdot\|\tilde{Y}\|}{A_{h}} \leqslant \frac{\|\mathcal{C}\|}{A_{h}} \cdot(2 \cdot\|\mathcal{M}\| \sqrt{N})=\frac{2 \cdot\|\mathcal{M}\| \cdot \sqrt{N}}{A_{h} /\|\mathcal{C}\|}
$$

where $\mathcal{C}=\left\langle C_{1}, \ldots, C_{L}\right\rangle$, and which shows that in order to minimize the influence of alien frequencies onto the value of phase $\varphi_{h}$ we need to minimize the value of $\|\mathcal{M}\| \sqrt{N}$. At the same time we see that the error on phase depends on the strength $\left(A_{h}\right)$ of the frequency component under discussion with comparison to the strength $(\|\mathcal{C}\|)$ of the alien part of the signal. 


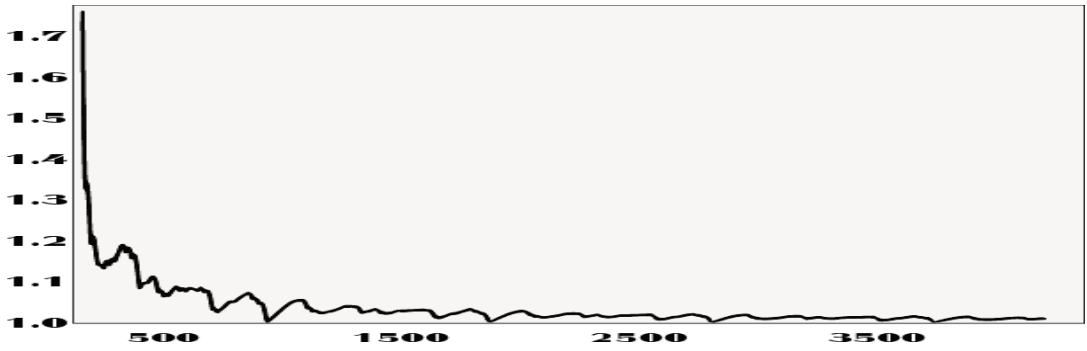

Fig. 2.4. Values of $\|\mathcal{M}\| \cdot \sqrt{N}$ as a function of signal length $N$

The Figure 2.4 shows a plot of $\|\mathcal{M}\| \cdot \sqrt{N}$ for frequencies $1.2,1.6$ and $2.1 \mathrm{MHz}$ with sampling $96 \mathrm{MHz}$.

Similarly as in the case of phase accuracy, we come across the following: PRoblem 2.

- For a given range of lengths, sampling frequency $F$ and $H$, find (quickly) a set/all sets of frequencies $f_{1}, \ldots, f_{H}$ with possibly small values of $\|\mathcal{M}\| \cdot \sqrt{N}$.

- Express analytically the correspondence

$$
\left\langle F, f_{1}, \ldots, f_{H}, N\right\rangle \mapsto\|\mathcal{M}\| \cdot \sqrt{N}
$$

- Find the best analytically expressible upper bound of $\|\mathcal{M}\| \cdot \sqrt{N}$.

\subsection{Unwinding}

As mentioned in the introduction, one possible way of recovering the evolution of phases is a suitable unwinding procedure which adjusts the observed sequence by an appropriate shift in order to avoid jumps which origin from periodical nature of phase. From the theoretical point of view the solution is quite easy. We have the following:

DEFINITION 3.

- The residual sequence for $\alpha \in{ }^{\mathbb{N}} \mathbb{R}$ is the sequence $\{\alpha\} \in{ }^{\mathbb{N}} \mathbb{R}$ such that

$$
\{\alpha\}_{n}=\left(\alpha_{n} \bmod 1\right), \quad n \in \mathbb{N}_{0}
$$


- The unfolding of $\alpha$ is a sequence $\widehat{\alpha}$ defined as

$$
(\widehat{\alpha})_{n}= \begin{cases}\alpha_{0} & n=0 \\ \alpha_{n}-\text { round }\left(\alpha_{n}-(\widehat{\alpha})_{n-1}\right) & n>0, \quad n \in \mathbb{N}_{0}\end{cases}
$$

Claim 4.

1. If $\alpha_{n} \in\langle 0,1), n \in \mathbb{N}$, then $\{\widehat{\alpha}\}=\alpha$.

2. If $\left|(\Delta \alpha)_{n}\right|<1 / 2, n \in \mathbb{N}$, then $\Delta(\widehat{\{\alpha\}}-\alpha)=0$.

Proof.

1. Let us first notice, that if $a \in\langle 0,1)$ and $N \in \mathbb{N}$ then

$$
a-N=\lfloor a-N\rfloor+\{a-N\}=\lfloor a\rfloor-N+\{a-N\}=-N+\{a-N\}
$$

hence $a=\{a-N\}$. Then also $\{\widehat{\alpha}\}_{0}=\left\{(\widehat{\alpha})_{0}\right\}=\left\{\alpha_{0}\right\}=\alpha_{0}$ and

$$
\{\widehat{\alpha}\}_{n}=\left\{(\widehat{\alpha})_{n}\right\}=\left\{\alpha_{n}-\operatorname{round}\left(\alpha_{n}-(\widehat{\alpha})_{n-1}\right)\right\}=\alpha_{n}
$$

for $n>0$,

2. We have:

$$
\begin{aligned}
& (\Delta(\widehat{\{\alpha\}}-\alpha))_{n}=(\widehat{\{\alpha\}}-\alpha)_{n+1}-(\widehat{\{\alpha\}}-\alpha)_{n}= \\
& \quad=(\widehat{\{\alpha\}})_{n+1}-\alpha_{n+1}-(\widehat{\{\alpha\}})_{n}+\alpha_{n}= \\
& \quad=\{\alpha\}_{n+1}-\operatorname{round}\left(\{\alpha\}_{n+1}-(\widehat{\{\alpha\}})_{n}\right)-\alpha_{n+1}-\{\alpha\}_{n}+\alpha_{n}= \\
& \quad=\{\alpha\}_{n+1}-\operatorname{round}\left(\{\alpha\}_{n+1}-\{\alpha\}_{n}\right)-\left(\left\lfloor\alpha_{n+1}\right\rfloor+\left\{\alpha_{n+1}\right\}\right)- \\
& \quad-\{\alpha\}_{n}+\left(\left\lfloor\alpha_{n}\right\rfloor+\left\{\alpha_{n}\right\}\right)= \\
& =-\operatorname{round}\left(\{\alpha\}_{n+1}-\{\alpha\}_{n}\right)-\left\lfloor\alpha_{n+1}\right\rfloor+\left\lfloor\alpha_{n}\right\rfloor= \\
& \quad=-\operatorname{round}\left(\{\alpha\}_{n+1}-\{\alpha\}_{n}+\left\lfloor\alpha_{n+1}\right\rfloor-\left\lfloor\alpha_{n}\right\rfloor\right)= \\
& \quad=-\operatorname{round}\left(\alpha_{n+1}-\alpha_{n}\right)=-\operatorname{round}\left((\Delta \alpha)_{n}\right)=0
\end{aligned}
$$

The problem is that the above works perfectly for sequencies without random distortions (see 2.5) but for sequencies obtained in real processes it sometimes gives expected results like in the Figure 2.6 but in many cases not (see 2.7). 

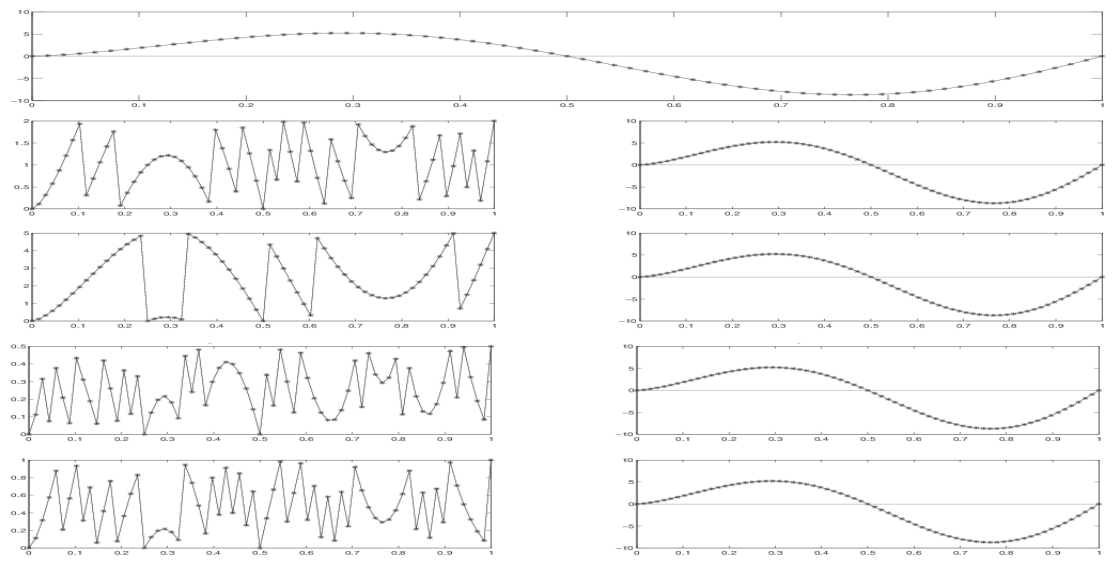

Fig. 2.5. At the top one sees a sample sequence and below to the left, the same sequence taken modulo 2.0, 5.0, 0.5 and 1.0, respectively. To the right there are the results of unwinding the sequence to the left.

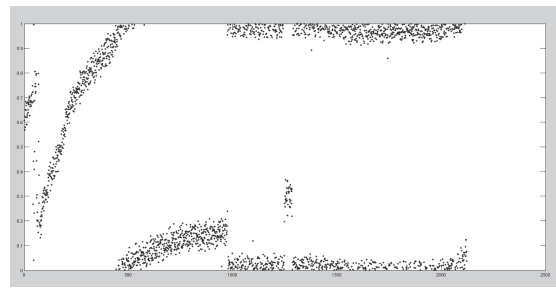

(a) Original phase time-series

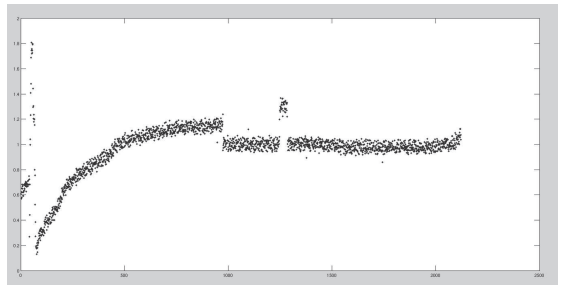

(b) Unwinding of the original time-series

Fig. 2.6. unwinding: correct result

Hence there arises the following:

Problem 5. Can one improve the unwinding so that it is more resistant to sudden momentary disturbances?

\subsection{Choice of Initial phases}

Because of digital nature of signals used in practice, one must avoid situations where there are to many regions in a signal with values significantly smaller then the values in other of them. In order to formalize the problem properly, we must introduce some notation first. 


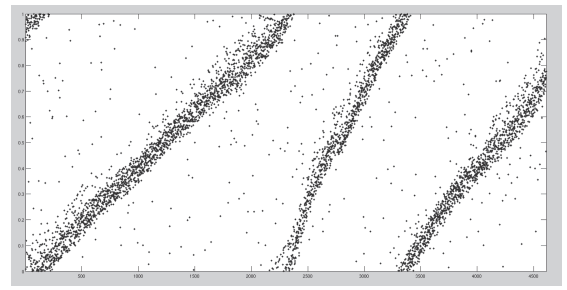

(a) Original phase time-series

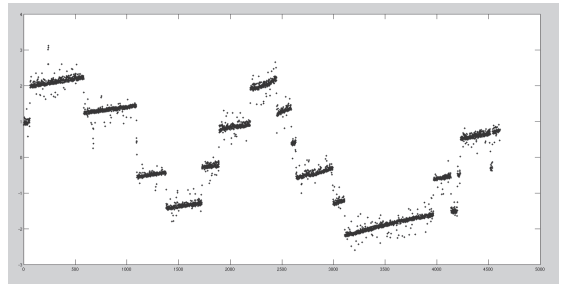

(b) Unwinding of the original time-series

Fig. 2.7. unwanted result of unwinding

Let $\mathcal{A}=\left\{A_{1}, \ldots, A_{H}\right\}, A_{1}, \ldots, A_{H} \in[0,1]$ be a set of amplitudes, $\mathcal{F}=\left\{F_{1}, \ldots, F_{H}\right\}$ a set of frequencies $\left(0<F_{h}<1 / 2, h=\right.$ $1, \ldots, H)$ and $\Psi=\left\{\psi_{1}, \ldots, \psi_{H}\right\}$ a set of unknown angles $\left(\psi_{h} \in\langle 0,2 \pi)\right.$, $h=1, \ldots, H)$. We consider a sequence $Y^{[\mathcal{A}, \mathcal{F}, \Psi]}=\left\langle Y_{n}\right\rangle_{n=0, \ldots, N-1}$ given as

$$
Y_{n}=\sum_{h=1}^{H} A_{h} \cdot \sin \left(2 \pi F_{h} \cdot n+\psi_{h}\right), \quad n=0, \ldots, N-1 .
$$
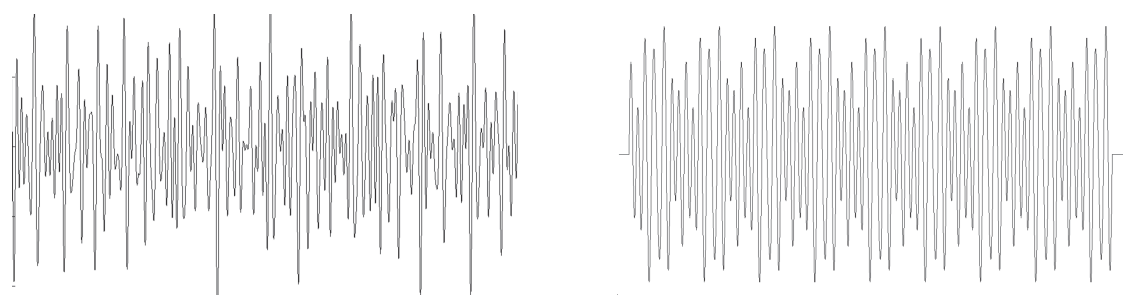

Fig. 2.8. Signals with improperly (to the left) and with properly distributed peaks (to the right)

Since the above has to be physically realizable, it must start from naught, which means that $\sum_{h=1}^{H} A_{h} \cdot \sin \left(\psi_{h}\right)=0$. On the other hand if $\Psi$ is not enough carefully chosen there can appear high peaks (see Figure 2.8) which after casting the signal to int16 numeric type cause frequencies with smaller amplitudes become to much rasterized which in turn may result in phase accuracy loss. That leads to the following: 
Problem 6. Find such a set $\Psi^{\mathrm{ini}}$ of initial phases which produces either of:

- The least possible hights of peaks in the signal. That is:

$$
\sup _{n}\left|Y_{n}^{\left[\mathcal{A}, \mathcal{F}, \Psi^{\mathrm{ini}}\right]}\right|=\inf _{\Psi} \sup _{n}\left|Y_{n}^{[\mathcal{A}, \mathcal{F}, \Psi]}\right| .
$$

- The least possible distance between maximal and minimal absolute values of local extremes in the signal:

$$
\frac{\min _{s}\left|\mu_{s}^{\left[\mathcal{A}, \mathcal{F}, \Psi^{\text {ini }}\right]}\right|}{\max _{s}\left|\mu_{s}^{\left[\mathcal{A}, \mathcal{F}, \Psi^{\mathrm{ini}}\right]}\right|}=\inf _{\Phi}\left(\frac{\min _{s}\left|\mu_{s}^{[\mathcal{A}, \mathcal{F}, \Psi]}\right|}{\max _{s}\left|\mu_{s}^{[\mathcal{A}, \mathcal{F}, \Psi]}\right|}\right)
$$

where $\mu_{s}^{\left[\mathcal{A}, \mathcal{F}, \Psi^{\text {ini }}\right]}$ is the absolute value of the $s^{\text {th }}$ peak of $Y^{[\mathcal{A}, \mathcal{F}, \Psi]}$.

Remark 7 . In the case one restricts themselves to the case $\psi_{h} \in\{0, \pi\}, h=$ $1, \ldots, H$, the "brute force" method requires $2^{H}$ steps. So even at least approximate solutions of the above are of interest.

\section{Conclusion and comments}

Acoustocerebrography is a new noinvasive method of monitoring and diagnosing a state of human's brain. We presented some problems of algebraic and/or algorithmic kind, we encountered during our research. Most of them hovewer were skipped since presenting them would overcome space limitations of this magazine. Amongst them are:

- Brunns differentional method for revealing frequencies

- Signal start estimation

- Unaliasing

- Phase curve tracking

Also some of the problems we mentioned in this paper may require reformulating in order to make them more precise. Some of the presented problems were given partial solutions. Complete solutions to these problems, however, still require a lot of effort. 


\section{References}

[1] M. Bogdan, et al., Computer Aided Multispectral Ultrasound Diagnostics Brain Health Monitoring System Based on Acoustocerebrography, XIV Mediterranean Conference on Medical and Biological Engineering and Computing 2016, IFMBE Proceedings Volume 57.

[2] M. O'Donnel, E. T. Jayess and J. G. Miller, Kramers-Kronig relationship between ultrasonic attenuation and phase velocity, J. Acoust. Soc. Am. 69(3), March 1981.

[3] J. Stoer, R. Bulirsch, Introduction to Numerical Analysis, Springer Science \& Business Media, March 20.

[4] M. Wrobel, et al., On ultrasound classification of stroke risk factors from randomly chosen respondents using non-invasive multispectral ultrasonic brain measurements and adaptive profiles, Biocybern Biomed Eng (2015), http://dx.doi.org/ 10.1016/j.bbe.2015.10.004.

e-mail: \{adam.kolany,miroslaw.wrobel\}@sonovum.de 